\section{INTEGRATED IMPLANT PLANNING}

Sirona offers an integrated concept consisting of 3D imaging and computer-aided implant planning and implementation. In combination the GALAXIS 3D X-ray software the GALILEOS system requires only one scan in order to visualise the entire cranial volume.

With the help of integrated software application GALILEOS Implant the

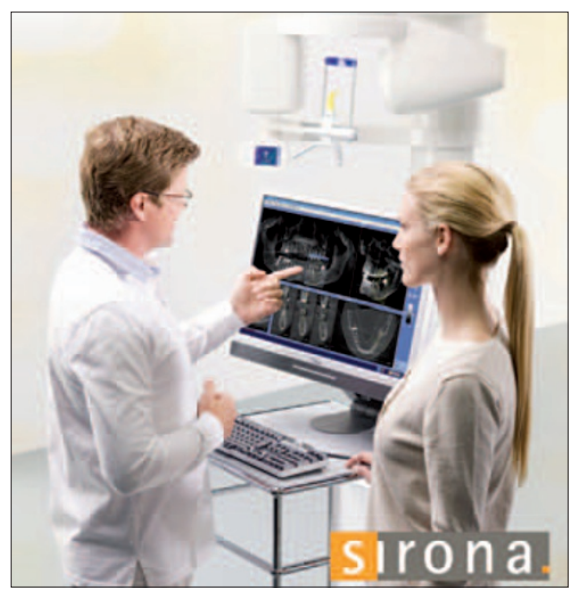

practitioner can plan the implants on the same user interface. This planning data, together with a plaster impression and bite registration, is then sent to the Sirona subsidiary siCAT in Bonn. In a matter of days the practitioner receives the custom-made surgical template.

The cone beam technology deployed in the GALILEOS system clearly reveals anatomical structures. Experimental images generated using the cone beam technique are just as accurate as images generated using computer tomography.

The Sirona cone beam technique is superior to conventional panoramic and cephalometric techniques with regard to the visualisation for the displaced wisdom teeth in the area of orthodontics.

In the field of cariology the images generated by the cone beam technique revealed diseased tooth tissue much more clearly than in the case with conventional X-rays.

Reader response number 54

\section{VITAL RESOURCE}

The Kodak 9000 3D from PracticeWorks is a model of innovation and affordability, providing a two-minute solution of both 2D panoramic and 3D imaging technology at the touch of a button to create a verifiable two in one solution for every day use.

Frequently a first step in examinations and treatments, panoramic images are required often in practice. Having the Kodak 9000 3D at your disposal will prove an invaluable resource.

Reader response number 55

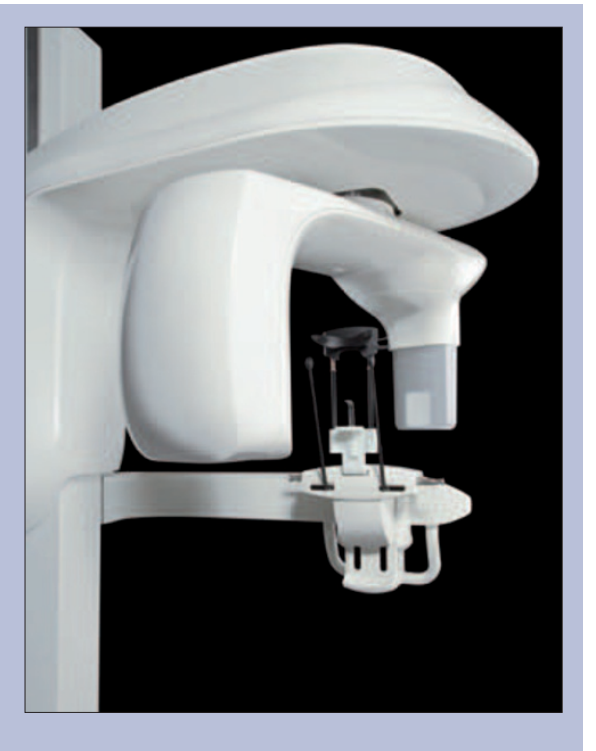

\title{
EFFECTIVE PRACTICE MANAGEMENT
}

With rising inflation, high cost of borrowing, decreasing exchange rates and general gloom now is the time to look at the value that DentalPlus practice management software offers. The DentalPlus licence fee is set at the point you join the system and never rises - not ever. Each year you benefit as inflation decreases the costs in real terms. Even better your licence to DentalPlus includes all new versions, all future developments, all help and assistance and daily offsite backups. Unlike other systems where they charge you now, then charge you again for new releases, upgrades and additional modules, DentalPlus includes all of these within its fixed-for-life annual licence charge. Make your accountant happy; predict your costs accurately into the future and share with others the benefits of being part of the DentalPlus community.

Reader response number 56

\section{CONSULTANCY SERVICES}

The Dental Practice Consultancy Service (DPCS) offers a range of services to help keep your practice running smoothly. These include practice valuation, sale and purchase, financial health check and practice income analysis and practice contractual agreements.

Financial analysis is crucial to make sure that your practice is functioning effectively. And if you are thinking of leaving the NHS, or implementing new charging schemes, let DPCS help you. We can provide expertise on partnership and associate agreements, PCT and GDS contracts and help with establishing or dissolving a practice.

Reader response number 57

\section{NEW DELIVERY UNIT}

The latest models of Trionic delivery units now include a redesigned touch control panel incorporating chair movements and programme selections, plus remote switching of the operating light. The new white finish adds to the appeal of these units.

Available in three formats: chair mounted, cabinet mounted or as a modern cart unit, all maintain the popular twin water bottle feature with remote switchover, also from the touch control panel.

There is ample space for the installation of a wide range of equipment options to meet individual surgeons' requirements. This attention to detail, together with ease of access for maintenance, and high build quality, make the Trionic units very popular. Reader response number 58

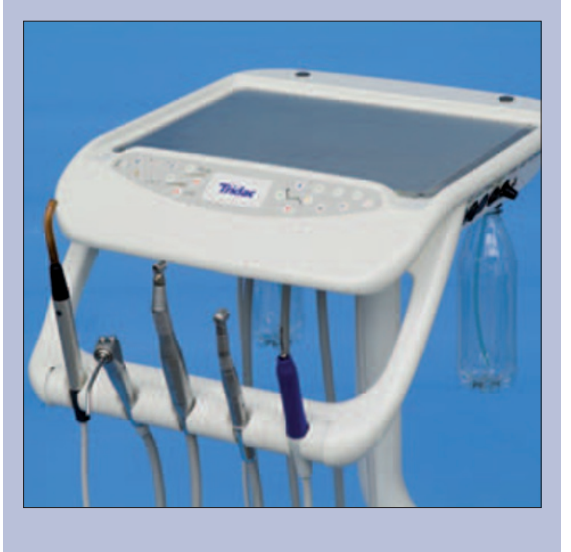

